\title{
GRIM-19 deficiency promotes clear cell renal cell carcinoma progression and is associated with high TNM stage and Fuhrman grade
}

\author{
NAIMENG YAN ${ }^{1 *}$, XUE FENG $^{1 *}$, SIXIONG JIANG ${ }^{2}$, \\ WEIBIN SUN ${ }^{2}$, MING-ZHONG SUN ${ }^{1}$ and SHUQING LIU ${ }^{1}$ \\ ${ }^{1}$ College of Basic Medicinal Sciences, Dalian Medical University, Dalian, Liaoning 116044; \\ ${ }^{2}$ Department of Urology, The Second Affiliated Hospital, Dalian Medical University, Dalian, Liaoning 116027, P.R. China
}

Received February 15, 2019; Accepted January 13, 2020

DOI: $10.3892 / \mathrm{ol} .2020 .11498$

\begin{abstract}
Clear cell renal cell carcinoma (ccRCC) exhibits the highest mortality among all urological malignancies. The investigation of the potential disease progression markers can improve ccRCC diagnosis and treatment. Gene associated with retinoid-interferon-induced mortality-19 (GRIM-19) is involved in carcinogenesis and cancer progression in a variety of cancer types including RCC. While, its role in ccRCC remains unclear, this cancer type is considered the most aggressive RCC subtype. In the present study, RT-qPCR, western blotting and immunohistochemical (IHC) assays demonstrated that GRIM-19 protein and mRNA levels were downregulated in ccRCC tumor tissues compared with the corresponding levels noted in paracancerous non-tumor tissues. The deficiency of this protein contributed in relaxed and/or collapsed structures of the kidney tubules and collecting duct noted in tumor tissues. Moreover, the reduction in GRIM-19 expression was associated with high tumor, lymph nodes and metastasis (TNM) stage and Fuhrman grade of ccRCC tumors. The data suggested that GRIM-19 acted as a tumor suppressor and that its deficiency promoted ccRCC development and progression. GRIM-19 can be considered a potential tumor marker for ccRCC.
\end{abstract}

\section{Introduction}

Renal cell carcinoma (RCC) accounts for approximately 3\% of all human malignancies and approximately $85 \%$ of renal

Correspondence to: Professor Shuqing Liu or Professor Ming-Zhong Sun, College of Basic Medicinal Sciences, Dalian Medical University, 9 Western Section, Lvshun South Road, Dalian, Liaoning 116044, P.R. China

E-mail: 1sqsmz@163.com

E-mail: smzlsq@163.com

*Contributed equally

Key words: gene associated with retinoid-interferon-induced mortality-19, clear cell renal cell carcinoma, development, progression cancer (1). Clear cell renal cell carcinoma (ccRCC) is responsible for approximately $80 \%$ of RCC incidence and exhibits the highest mortality rate among all urological malignancies $(2,3)$. The 5-year survival rate of a major percentage of patients diagnosed with metastatic ccRCC (>30\%) was below $20 \%$ (4). The successful treatment for ccRCC, notably for metastatic ccRCC, is becoming increasingly challenging for medical practitioners $(5,6)$. The discovery of novel tumor markers can improve the understanding, diagnosis and treatment of ccRCC.

Gene associated with retinoid-interferon-induced mortality-19 (GRIM-19) is a member of the GRIM family of proteins $(7,8)$. It is essential for the tumor cell death induced by interferon- $\beta$ and retinoic acid. As a subunit of mitochondrial NADH: ubiquinone oxidoreductase (respiratory chain complex I), GRIM-19 is critical for complex I assembly, activity and mitochondrial membrane potential maintenance $(9,10)$. Its deficiency or mutation commonly increases the sensitivity of the cells to their apoptotic stimuli $(9,10)$. GRIM-19 is well-known as a cell death regulatory gene widely distributed in the cytoplasm, nucleus and mitochondria and has been studied as a suppressor of various types of cancer (11-17). GRIM-19 downregulation is associated with the development, invasion and induction of apoptosis of lung cancer (11), glioma (12), cervical cancer (13), breast cancer (14), hepatocarcinoma $(15,16)$ and gastric cancer cells $(17)$. GRIM-19 may be considered an important tumor marker.

The present study analyzed the mRNA and protein expression levels and distribution of GRIM-19 in ccRCC tumor tissues compared with those noted in the paracancerous non-tumor tissues, and demonstrated that GRIM-19 deficiency promoted ccRCC malignant progression by causing an increase in the tumor, lymph nodes and metastasis (TNM) stage and the Fuhrman grade of the tumors.

\section{Materials and methods}

Specimen collection from ccRCC patients. A total of 93 paired-matched tumor and paracancerous non-tumor renal tissues were obtained from ccRCC patients during the period June 2015 and October 2016 at the Second Affiliated Hospital of the Dalian Medical University, in Dalian, Liaoning, China. 
Non-tumor renal tissues were obtained from a distance higher than and/or equal to $5(\geq 5) \mathrm{cm}$ away from the edge of the resected tumor. None of the patients received radiotherapy or chemotherapy prior to surgery. The specimens were classified by the patient clinicopathological parameters including age, gender, tumor position, TNM stage and Fuhrman grade. A total of 74 and 19 paired tissues were from male and female patients, whereas 43 and 50 paired tissues were from patients higher than $60(>60)$ and lower than and/or equal to $60(\leq 60)$ years, respectively. A total of 51 and 42 paired tissues were obtained from the right and left side kidneys of the patients, respectively. A total of 54, 16, 22 and 1 tumors were of $\mathrm{T} 1$, T2, T3 and T4 stage, respectively, whereas 53 and 40 tumors were of Fuhrman grade $\leq 2$ and Fuhrman grade $>2$. The histological subtypes and tumor stages were assessed by the 2016 WHO Classification of Tumors of the Urinary System of the Tumor, Nodes and Metastasis system (18). A total of 38 cases of paired surgical tissues were snap-frozen in liquid nitrogen for RT-qPCR and western blotting analyses, whereas 55 paired tissues were fixed in $10 \%$ neutral-buffered formalin for $24 \mathrm{~h}$ at room temperature (RT) and embedded in paraffin for the immunohistochemical (IHC) assay.

$R T$ - $q P C R$ assay. A piece of tissue $(\sim 50 \mathrm{mg})$ from each patient was cut into $50 \mu \mathrm{m}$ slices by a freezing microtome. Total RNA was extracted using TRIzol reagent (Transgen Biotech Co., Ltd.) and reversely transcribed into cDNA using EasyScript One-Step gDNA Removal and cDNA Synthesis SuperMix kit (Transgen Biotech Co., Ltd.). RT-qPCR was performed using Quantitect SYBR Green PCR kit (Transgen Biotech Co., Ltd.) in a StepOnePlus PCR system (Thermo Scientific, Inc.). The following conditions were used: Initial denaturation at $94^{\circ} \mathrm{C}$ for $30 \mathrm{sec}$, followed by 45 cycles at $94^{\circ} \mathrm{C}$ for $5 \mathrm{sec}, 60^{\circ} \mathrm{C}$ for $30 \mathrm{sec}, 95^{\circ} \mathrm{C}$ for $15 \mathrm{sec}, 60^{\circ} \mathrm{C}$ for $60 \mathrm{sec}, 95^{\circ} \mathrm{C}$ for $30 \mathrm{sec}$ and $60^{\circ} \mathrm{C}$ for $30 \mathrm{sec}$. GAPDH was used as the internal reference gene. The primer sequences for GRIM-19 were the following: F: 5'-ACCGGAAGTGTGGGATACTG-3', R: 5'-GCTCAC GGTTCCACTTCATT-3' and for GAPDH F: 5'-AGAAGG CTGGGGCTCATTTG-3', R: 5'-AGGGGCCATCCACAGTC TTC-3'. The $2^{-\triangle \Delta C T}$ method was used for quantification (19). GRIM-19 level in the tumorous tissue from one ccRCC patient was strictly compared with and normalized against its level that was defined as $1(100 \%)$ in paired paracancerous normal tissue from same patient. The amplification cycle times of the internal reference in paired tumor and paracancerous tissues were separately used for calculating the relative differential cycle time $(\triangle \mathrm{CT})$ and relative differential expression level change $(\Delta \Delta \mathrm{CT})$ of GRIM-19. The overall difference of GRIM-19 in ccRCC patients' tumorous tissues with $\mathrm{P}$ below 0.05 was considered statistically significant.

Western blotting. Approximately $50 \mathrm{mg}$ of tissue from each patient was washed with $\mathrm{PBS}$, grounded in liquid nitrogen in a mortar covered with aluminum foil into powder and suspended in $450 \mu \mathrm{l}$ of ice-cold RIPA solution. The tissue mixture was grounded using a pestle and suspended thrice for 10 min each time on ice. The total protein was collected from the supernatant by centrifugation at $12,879 \times \mathrm{g}$ at $4^{\circ} \mathrm{C}$ for $15 \mathrm{~min}$. The protein concentration was determined by the Bradford assay. Equal amounts of protein from each group were boiled for 5 min and separated by PAGE using 10\% SDS gels. The protein bands were transferred onto nitrocellulose membranes (PALL Co., Ltd.), blocked in 5\% (w/v) skimmed milk (Becton, Dickinson) in TBST for $3 \mathrm{~h}$ at RT and incubated with primary antibodies against GRIM-19 (1:1,000; 10986-1-AP; Proteintech Group, Inc.) and GAPDH (1:2,000; 10494-1-AP; Proteintech Group, Inc.) at $4^{\circ} \mathrm{C}$ overnight. The membranes were washed with TBST thrice for $10 \mathrm{~min}$ each time, incubated with the secondary antibody $\operatorname{IgG}(1: 2,000$; SA00001-2; Proteintech Group, Inc.) for $3 \mathrm{~h}$ at RT, and washed with TBST for an additional three times (10 min each time). The protein bands were visualized by ECL (K-12045-D50; Advansta) and analyzed by the Bio-Rad ChemiDoc ${ }^{\mathrm{TM}} \mathrm{MP}$ system (Bio-Rad, Laboratories, Inc.). GRIM-19 protein expression level in the tumorous tissue from one ccRCC patient was strictly compared with and normalized against its level that was defined as $1(100 \%)$ in paired paracancerous normal tissue from same patient. Its level change with P-value less than 0.05 was regarded as statistically significant.

IHC assay. Paraffin-embedded tumor and non-tumor renal tissues were cut into 4- $\mu \mathrm{m}$ slices. The slides were boiled in citrate buffer in a micro-wave oven with high power for 4 min and cooled down at RT. This step was repeated four times. Following washing with PBS three times for 5 min each time, the slices were blocked in $3 \% \mathrm{H}_{2} \mathrm{O}_{2}$ for $20 \mathrm{~min}$ and in $10 \%$ non-immune goat serum (ZSGB-BIO, Co., China) for $15 \mathrm{~min}$ at RT. The samples were incubated with GRIM-19 antibody $(1: 200)$ at $4^{\circ} \mathrm{C}$ overnight, placed at $37^{\circ} \mathrm{C}$ for $30 \mathrm{~min}$ and treated with biotin-streptavidin HRP detection kit (ZSGB-BIO, Co.). The images were developed using 3,3'-diamino-benzidine kit (ZSGB-BIO, Co.) under an upright light BX3-CBH microscope (Olympus, Co.).

The classification of the IHC staining was assessed by the immunoreaction intensity and the DAB positive staining quantity of the tumor cells (20). The immunoreaction intensity was classified as 0 (negative), 1 (weak), 2 (moderate) and 3 (strong). The DAB staining quantity of each sample was rated as 0 (none), 1 (1-10\% positive cells/field), 2 (10-50\%), 3 (51-75\%) and $4(>76 \%)$. Their multiplication scores ranged from $0-2$, $3-5,6-8$ and 9-12, and were considered as negative (-), weak $(+)$, moderate $(++)$ and strong $(+++)$ with regard to GRIM-19 expression levels, respectively.

Data processing and statistical analysis. Experimental data were expressed as mean \pm standard deviation. The data were processed by SPSS 17.0 Software (SPSS, Inc.). The differential expression between tumor and paired non-tumor tissues was analyzed by the paired t-test and the one-way ANOVA (and nonparametric) analysis with the post hoc Brown-Forsythe test. The expression levels of GRIM-19 were compared with the patient clinicopathological parameters by the $\chi^{2}$ or Fisher's exact tests. Significant differences were considered for $\mathrm{P}<0.05$.

\section{Results}

GRIM-19 mRNA downregulation is associated with ccRCC clinical progression. The alterations in GRIM-19 mRNA levels were evaluated in paired tumor and paracancerous non-tumor tissues from 38 patients by RT-qPCR. GRIM-19 
Table I. Clinical association of GRIM-19 mRNA and protein alterations with clear cell renal cell carcinoma.

\begin{tabular}{|c|c|c|c|c|c|}
\hline \multirow[b]{2}{*}{ Parameter } & \multirow[b]{2}{*}{ Patients, $\mathrm{n}$} & \multicolumn{4}{|c|}{ Level changes and clinical significance } \\
\hline & & Relative mRNA level $^{a}$ & P-value & Relative protein level $^{\mathrm{a}}$ & P-value \\
\hline Sex & & & 0.300 & & 0.591 \\
\hline Male & 31 & 0.715 & & 0.231 & \\
\hline Female & 7 & 0.466 & & 0.119 & \\
\hline Age, years & & & 0.073 & & 0.293 \\
\hline$\leq 60$ & 22 & 0.834 & & 0.192 & \\
\hline$>60$ & 16 & 0.443 & & 0.116 & \\
\hline Tumor location & & & 0.129 & & 0.541 \\
\hline Left kidney & 17 & 0.825 & & 0.241 & \\
\hline Right kidney & 21 & 0.543 & & 0.119 & \\
\hline T stage & & & 0.023 & & 0.003 \\
\hline pT1 & 23 & 0.809 & & 0.200 & \\
\hline pT2 & 7 & 0.605 & & 0.089 & \\
\hline pT3-4 ${ }^{\mathrm{b}}$ & 8 & 0.285 & & 0.033 & \\
\hline Fuhrman grade & & & 0.035 & & 0.009 \\
\hline$\leq 2^{\mathrm{c}}$ & 20 & 0.836 & & 0.197 & \\
\hline$>2^{\mathrm{d}}$ & 18 & 0.485 & & 0.086 & \\
\hline
\end{tabular}

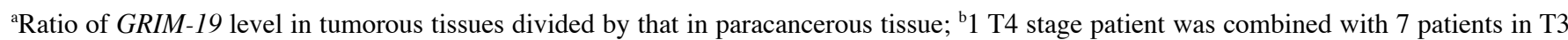
stage; ${ }^{c} 1$ patient in Fuhrman grade 1 was combined with 19 patients in grade 2; ${ }^{\mathrm{d}} 1$ patient in Fuhrman grade 4 was combined with 17 patients in grade 3. GRIM-19, gene associated with retinoid-interferon-induced mortality-19.
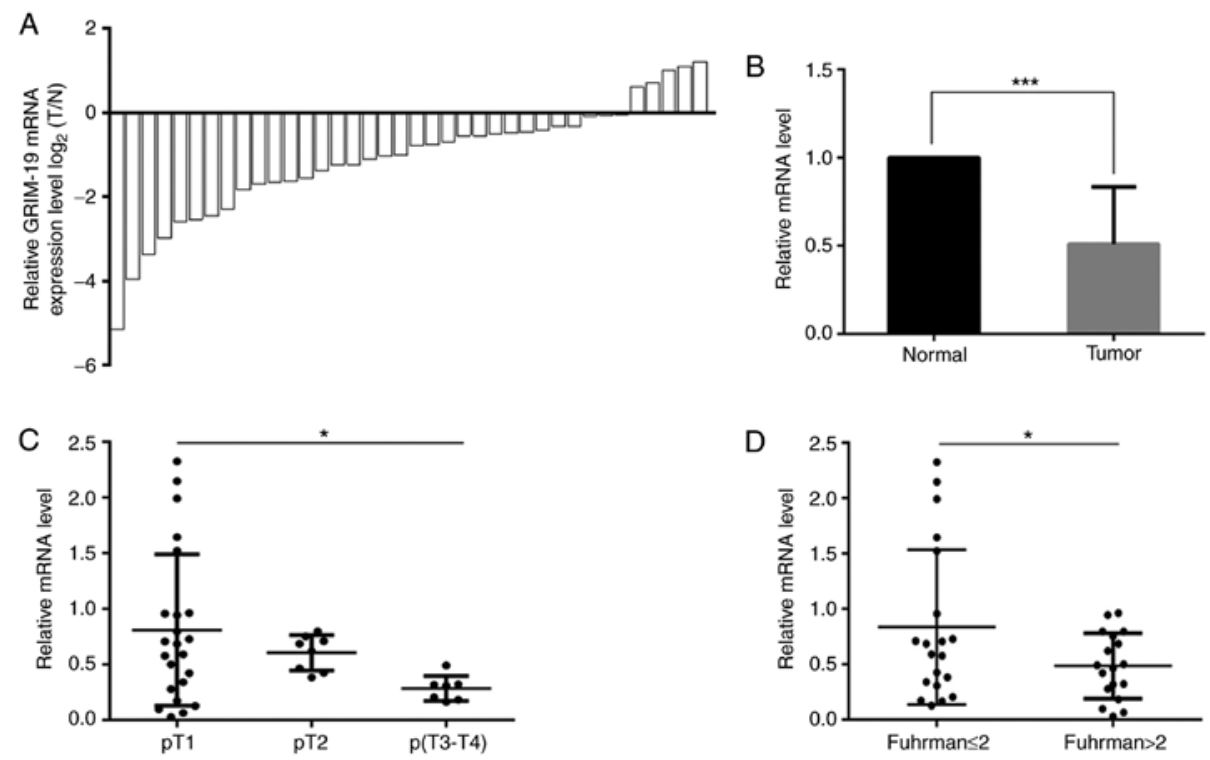

Figure 1. Decreased GRIM-19 mRNA levels are inversely associated with ccRCC progression. (A) Reverse transcription-quantitative PCR assay of the GRIM-19 levels in tumor and paired paracancerous non-tumor renal tissues from 38 patients. (B) GRIM-19 levels were decreased in tumor tissues (P=0.0007). GRIM-19 level in tumorous tissue from one patient with ccRCC was strictly compared with and normalized against its level that was defined as 1 (100\%) in paired paracancerous normal tissue from the same patient. GRIM-19 downregulation was associated with (C) high TNM stage (P=0.02) and (D) the Fuhrman grade (P=0.03) of the tumors. ${ }^{*} \mathrm{P}<0.05$ and ${ }^{* * *} \mathrm{P}<0.001$. GRIM-19, gene associated with retinoid-interferon-induced mortality-19; ccRCC, clear cell renal cell carcinoma.

was downregulated in the majority $(33 / 38)$ of the tumor tissues (Fig. 1A) with an overall decrease of $49.1 \%(\mathrm{P}=0.0007$, Fig. 1B). GRIM-19 downregulation was inversely associated with the $\mathrm{T}$ stage and the Fuhrman grade of the tumors, while it was not associated with the patient age $(\mathrm{P}=0.07)$, gender
$(\mathrm{P}=0.3)$ and tumor location (tumor from left or right kidney, $\mathrm{P}=0.1$, Table I). The GRIM-19 levels noted in the T2 and T3-T4 tumor tissues were decreased by 25.2 and $64.8 \%$, respectively compared with those noted in the $\mathrm{T} 1$ stage samples (Fig. 1C, Table I, $\mathrm{P}=0.02)$. GRIM-19 levels in tumors with Fuhrman 

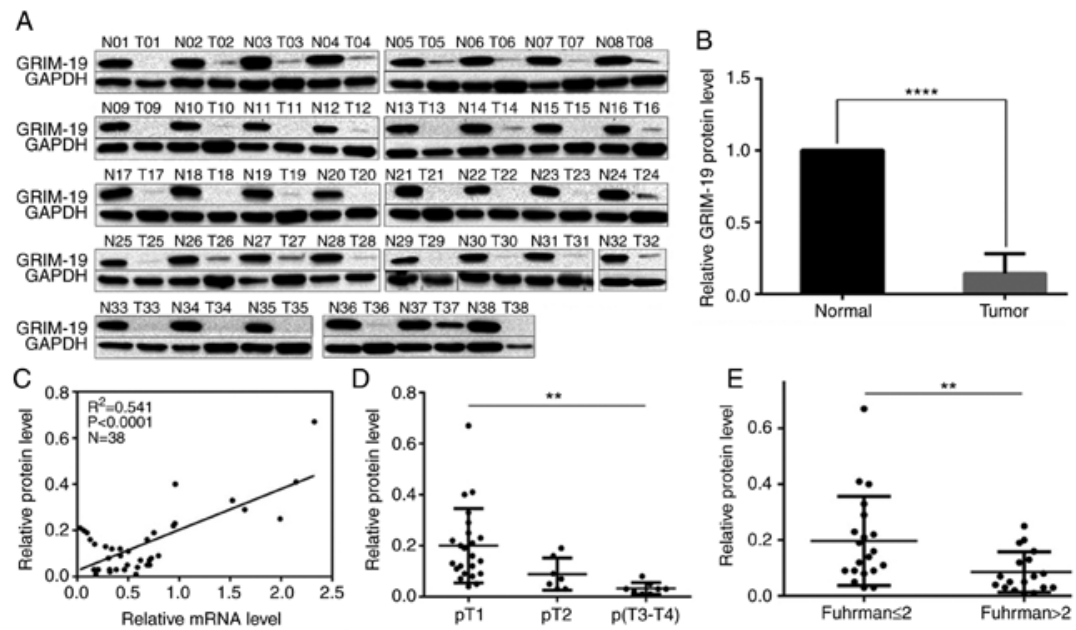

Figure 2. Downregulation of GRIM-19 protein levels enhances ccRCC progression. (A) Western blotting of GRIM-19 levels in tumor tissues and paired paracancerous non-tumor tissues from 38 patients. There appears to be a break in continuity between the GAPDH bands obtained from samples N29 and T31. The GAPDH band from N29 was shown to the upper left on gel, a slight rotation and adjustment was made for it together with T29 for a better plot view. The GRIM-19 bands from N32 and T32 were obtained from the same gel running for N29-N32 and T29-T32. The GAPDH bands from N32 and T32 were obtained from another gel running as they were not visualized on the same gel for N29-N32 and T29-T32. (B) GRIM-19 levels were decreased by $85.6 \%$ in ccRCC tumor tissues $(\mathrm{P}=0.0001)$. GRIM-19 level in tumorous tissue from one ccRCC patient was strictly compared with and normalized against its level that was defined as $1(100 \%)$ in paired paracancerous normal tissue from the same patient. (C) Downregulation of GRIM-19 mRNA levels was positively correlated with the downregulation of the GRIM-19 protein expression levels $\left(\mathrm{R}^{2}=0.541 ; \mathrm{P}<0.0001\right)$. GRIM-19 protein downregulation was associated with (D) high TNM stage $(\mathrm{P}=0.003)$ and $(\mathrm{E})$ Fuhrman grade $(\mathrm{P}=0.009) .{ }^{* *} \mathrm{P}<0.01$ and $^{* * * * *} \mathrm{P}<0.0001$. GRIM-19, gene associated with retinoid-interferon-induced mortality-19; ccRCC, clear cell renal cell carcinoma.

grade lower than and/or equal to $2(\leq 2)$ were $72.4 \%$ higher than those of tumors with Fuhrman grade higher than 2 (>2, Fig. 1D, Table I, $\mathrm{P}=0.03$ ). These findings suggested that GRIM-19 deficiency promoted ccRCC progression.

GRIM-19 protein downregulation in tumor tissues exhibits a negative correlation with ccRCC clinical aggressiveness. Western blotting indicated that GRIM-19 protein levels were ubiquitously suppressed in each tumor tissue compared with the corresponding paired non-tumor tissues (Fig. 2A). GRIM-19 levels in tumor tissues increased from 1 to $67 \%$ compared with those in paired non-tumor tissues (Fig 2B, $\mathrm{P}=0.0001$ ). An overall decrease of approximately $85.6 \%$ was noted (Fig. 2B, $\mathrm{P}=0.0001)$. A positive correlation was noted between GRIM-19 protein and mRNA downregulation (Fig. $2 \mathrm{C}, \mathrm{P}<0.0001$ ). The downregulation noted in the protein levels was inversely associated with the increase in the tumor stage and in the Fuhrman grade (Table I). GRIM-19 protein levels were decreased by 55.0 and $83.5 \%$ in T2 and T3-4 tumors, respectively compared with the levels noted in T1 tumors (Fig. 2D, Table I, $\mathrm{P}=0.003$ ). GRIM-19 protein levels were decreased by $56.4 \%$ in tumors with Fuhrman grade higher than $2(>2)$ compared with those of tumors with Fuhrman grade lower than and/or equal to 2 ( $\leq$ 2) (Fig. 2E, Table I, $\mathrm{P}=0.009$ ). This evidence was consistent with the aforementioned findings. No significant differences were noted with regard to the reduction in the GRIM-19 protein expression levels measured in tumor tissues from female and male patients (48.5\% reduction, Table I, $\mathrm{P}=0.591$ ). Similar findings were noted in the tissues from patients higher and lower than, and/or equal to 60 years ( $>60$ or $\leq 60$ years) (39.6\% lower, Table I, $\mathrm{P}=0.293$ ). Furthermore, no significant differences were noted with regard to the reduction in the GRIM-19 protein expression levels in tissues from patients
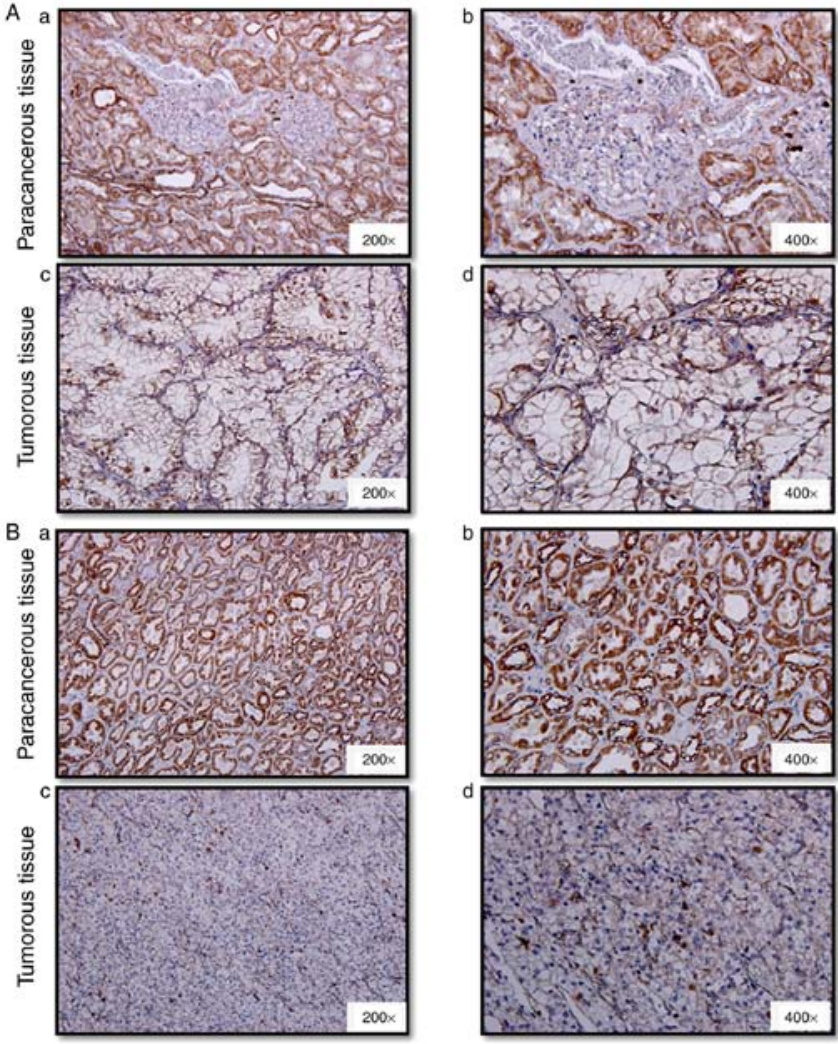

Figure 3. Immunohistochemistry evaluation of GRIM-19 levels and protein distribution in ccRCC tumor and paracancerous tissues. Representative images of GRIM-19 expression in the paracancerous region, (Aa and Ab) and the tumor region (well-differentiated, Ac and Ad) from one tumor sample of Fuhrman grade 2; Paracancerous tissues $(\mathrm{Ba}$ and $\mathrm{Bb}$ ) and tumor tissues (poorly differentiated, $\mathrm{Bc}$ and $\mathrm{Bd}$ ) from one patient with Fuhrman grade 3. The images corresponding to $\mathrm{Aa}, \mathrm{Ac}, \mathrm{Ba}$ and $\mathrm{Bc}$, and to $\mathrm{Ab}, \mathrm{Ad}, \mathrm{Bb}$ and $\mathrm{Bd}$ were obtained at x200 and x400 magnification, respectively. GRIM-19, gene associated with retinoid-interferon-induced mortality-19; ccRCC, clear cell renal cell carcinoma. 
Table II. Immunohistochemistry assay of GRIM-19 association with clear cell renal cell carcinoma clinicopathology parameters.

GRIM-19 positive immunoreactivity

\begin{tabular}{|c|c|c|c|c|}
\hline \multirow[b]{2}{*}{ Parameter } & \multirow[b]{2}{*}{ Specimen, $n(n=55)$} & \\
\hline & & Positive, $\mathrm{n}(\%)(\mathrm{n}=21)$ & Negative, $n(\%)(n=34)$ & P-value \\
\hline Sex & & & & 0.750 \\
\hline Male & 43 & $17(81.0)$ & $26(76.5)$ & \\
\hline Female & 12 & $4(19.0)$ & $8(23.5)$ & \\
\hline Age, years & & & & 0.269 \\
\hline$\leq 60$ & 28 & $13(61.9)$ & $15(44.1)$ & \\
\hline$>60$ & 27 & $8(38.1)$ & $19(55.9)$ & \\
\hline Tumor position & & & & 0.610 \\
\hline Left kidney & 34 & $13(61.9)$ & $21(61.8)$ & \\
\hline Right kidney & 21 & $8(38.1)$ & $13(38.2)$ & \\
\hline T stage & & & & 0.013 \\
\hline $\mathrm{T} 1-\mathrm{T} 2$ & 40 & $19(90.5)$ & $21(61.8)$ & \\
\hline $\mathrm{T} 3$ & 15 & $2(9.5)$ & $13(38.2)$ & \\
\hline Fuhrman grade & & & & 0.026 \\
\hline$\leq 2$ & 33 & $17(81.0)$ & $16(47.1)$ & \\
\hline$>2$ & 22 & $4(19.0)$ & $18(52.9)$ & \\
\hline
\end{tabular}

GRIM-19, gene associated with retinoid-interferon-induced mortality-19.

with right and left kidneys (50.6\% lower, Table I, $\mathrm{P}=0.541)$. The downregulation of the mRNA and protein levels of GRIM-19 was concordantly associated with ccRCC aggressiveness.

The expression levels and protein distribution of GRIM-19 are associated with ccRCC clinical characteristics. GRIM-19 levels and protein localization in patient tumor and paracancerous non-tumor tissues were analyzed by immunohistochemistry. The expression of GRIM-19 was mainly present in the cytoplasm (Fig. 3). GRIM-19 levels were highly elevated in normal kidney epithelial cells, tubular epithelial cells and collecting duct epithelial cells (Figs 3-1A-B, 2A-B). This protein was not expressed in the nuclei of kidney glomerular cells (Fig. 3). The following immunoreactivity scores were noted in $61.8 \%(34 / 55), 27.3 \%(15 / 55), 5.45 \%(3 / 55)$ and $5.45 \%(3 / 55)$ of tumor tissues:,,-+++ and +++ . The same immunoreactivity expression pattern $(-,+,++$ and +++$)$ was noted in $0 \%(0 / 40), 25 \%(10 / 40), 35 \%(14 / 40)$ and $40 \%(16 / 40)$ of paracancerous non-tumor tissues (Table II, $\mathrm{P}<0.0001)$. GRIM-19 was undetected in 61.8\% (34/55) of tumor tissues, while, it was present in all (40/40) the paracancerous tissues. The percentage of tumor tissues that exhibited ++ and +++ GRIM-19 immunoreactivity was $10.9 \%$ (6/55), which was $64.1 \%$ lower than that of the paracancerous non-tumor tissues (75\%, 30/40). GRIM-19 expression levels were downregulated or undetectable in tumor tissues compared with those noted in paracancerous tissues (Figs 3-1C-D, 2C-D, Figs 3-1A-B, 2A-B). GRIM-19 levels were decreased or undetectable in tissues that exhibited higher tumor stage compared with the corresponding levels noted in low stage tumor tissues (Figs 3-1C-D, 3-2C-D). Accordingly, the structural arrangements of the kidney tubules and collecting duct became loose (Fig. 3-1C-D) or collapsed
(Fig. 3-2C-D). Further analysis indicated that GRIM-19 downregulation was inversely associated with the increase in the tumor stage $(\mathrm{P}=0.013)$ and in the tumor Fuhrman grade $(\mathrm{P}=0.026)$, while it was not associated with the patient gender, age and tumor location (Table II). These findings were consistent with the RT-qPCR and Western blotting results.

In conclusion, the present study explored the dysregulation in GRIM-19 expression levels with the clinical development and progression of ccRCC by RT-qPCR, Western blotting and IHC assays. GRIM-19 acted as a tumor suppressor in ccRCC. Its deficiency was associated with an increase in the tumor stage and in the Fuhrman tumor grade of ccRCC.

\section{Discussion}

The contribution of GRIM-19 in the regulation of tumor growth has been recently recognized. GRIM-19 is commonly regarded as a tumor suppressor in certain cancer types. The suppression of GRIM-19 expression promoted lung cancer metastasis (11) and enhanced glioma cell proliferation and migration (12). Accordingly, GRIM-19 overexpression suppressed cell proliferation of cervical cancer, breast cancer, glioma, hepatocarcinoma and gastric cancer by inducing the apoptotic cascade (12-17). This protein may be considered an important tumor marker. Proteomic analysis indicated that GRIM-19 was downregulated in RCC (21). A recent study highlighted that GRIM-19 was downregulated in RCC tissues and that it could inhibit tumor growth by decreasing cell proliferation and inducing apoptosis (22). The present study was specifically designed to explore the contribution of GRIM-19 deficiency in the clinical progression of ccRCC, which is considered the most severe type of RCC. 
The results suggested that GRIM-19 mRNA and protein levels were lower in tumor tissues from ccRCC patients compared with the levels noted in the paired paracancerous non-tumor renal tissues. The findings were similar to those noted from other reports that confirmed that the expression levels of GRIM-19 were reduced or totally suppressed in a number of human tumor types, such as lung cancer, cervical cancer, hepatocarcinoma, gastric cancer and glioma. GRIM-19 deficiency was associated with poor differentiation, lymph node metastasis and vascular invasion of colorectal cancer (23). Moreover, GRIM-19 deficiency enhanced the progression of lung cancer (11) and glioma (12). Its downregulation led to high clinical stage, increased volume of ascites and increased size of primary tumor lesion, while it was also associated with reduced survival rate of epithelial ovarian carcinoma patients (24). Downregulation of GRIM-19 closely correlated with high histological grade in HCC. The association of GRIM-19 levels with the clinicopathological features of ccRCC is unclear. The present study demonstrated that GRIM-19 deficiency was associated with high TNM stage and Fuhrman grade of ccRCC tissues.

GRIM-19 expression is differentially distributed in various organelles depending on the tumor cell and tissue type. It was expressed mainly in the nucleus of HeLa cells (13) and in the cytoplasm of hepatocarcinoma cells (15). Fan et al (11) demonstrated that GRIM-19 was primarily localized in the cytoplasm of inflamed lung tissues, whereas it demonstrated nuclear distribution in the nuclei of lung cancer cells. GRIM-19 was only detected in the cytoplasm of colorectal cancer cells compared with its expression in the corresponding normal cells, where it was localized in both cytoplasmic and nuclear regions (23). The present study indicated that GRIM-19 was highly expressed in normal kidney epithelial, renal tubular epithelial and collecting duct epithelial cells, while it was not expressed in the nuclei and glomerular cells of paracancerous tissues. GRIM-19 expression levels were undetectable in tumor tissues compared with those noted in paracancerous tissues. The loss or reduction of GRIM-19 was more evident in tumor tissues of higher stage than tumor tissues of low stage, which resulted in enhanced structural loss or collapse of the kidney tubules and the collecting duct.

Signal transducer and activator of transcription 3 (STAT3) is a latent cytoplasmic transcription factor that plays important roles in cell growth, reduction of apoptosis and cell transformation. Accumulating evidence has suggested that GRIM-19 is a negative regulator of STAT3, repressing STAT3 transcriptional activity and its downstream target gene expression. In human cervical cancer, the restoration of GRIM-19 levels reestablishes the control over STAT3-dependent gene expression and affects tumor growth in vivo (13). The downregulation of GRIM-19 expression correlates with hyperactivation of STAT3 in HCC (15). GRIM-19 expression sensitizes BGC-803 gastric cancer cells to radiation possibly via the suppression of STAT3 accumulation (17). Overexpression of GRIM-19 is associated with hyperactivation of STAT3-induced gene expression in renal carcinoma (22). Downregulation of GRIM-19 correlated with STAT3 overexpression in tumor samples from breast cancer patients (25). This finding provides additional evidence for unraveling the exact mechanism of action of GRIM-19 in ccRCC.

In summary, the present study suggests that GRIM-19 acts as a tumor suppressor in ccRCC. Its deficiency promotes
ccRCC aggressiveness. The overall GRIM-19 levels were dramatically decreased and exerted an effect on the relaxed and/or collapsed structures of the kidney tubules and the collecting duct in the tumor tissues from ccRCC patients. GRIM-19 deficiency is a potential indicator for ccRCC malignant transformation.

In future study on GRIM-19, we will follow up on the lifespan of the patients. and consider for getting the tissue array of ccRCC patients with survival times and rates. At the same time, we will investigate the association of GRIM-19 deficiency with the malignant progression, especially, the survival time for ccRCC patients. Surely enough, more investigations are worth doing for validating and confirming the role of GRIM-19 as a reliable biomarker, surgical and therapeutic target in clinical significance for ccRCC.

\section{Acknowledgements}

Not applicable.

\section{Funding}

Thisstudy wassupportedbygrants from NationalNaturalScience Foundation of China (grant nos. 81672737 and 81272186).

\section{Availability of data and materials}

The datasets used and/or analyzed during the current study are available from the corresponding author on reasonable request.

\section{Authors' contributions}

$\mathrm{NY}$ and XF performed the experiments and wrote manuscript draft. SJ collected the specimens and extracted proteins from specimens. WS designed the experiment and validated clinicopathological diagnosis of patients. MZS and SL designed and supervised the study and corrected the manuscript.

\section{Ethics approval and consent to participate}

Tissue use and the study protocol were approved by the Medical Ethics Committee of Dalian Medical University, and granted by the informed consent from all patients.

\section{Patient consent for publication}

Written informed consents were obtained from all individual participants.

\section{Competing interests}

The authors declare that they have no competing interests.

\section{References}

1. Barata PC and Rini BI: Treatment of renal cell carcinoma: Current status and future directions. CA Cancer J Clin 67: 507-524, 2017.

2. De Meerleer G, Khoo V, Escudier B, Joniau S, Bossi A, Ost P, Briganti A, Fonteyne V, Van Vulpen M, Lumen N, et al: Radiotherapy for renal-cell carcinoma. Lancet Oncol 15: e170-e177, 2014. 
3. Oosterwijk E, Rathmell WK, Junker K, Brannon AR, Pouliot F, Finley DS, Mulders PF, Kirkali Z, Uemura $\mathrm{H}$ and Belldegrun A: Basic research in kidney cancer. Eur Urol 60: 622-633, 2011.

4. Ficarra V, Guillè F, Schips L, de la Taille A, Prayer Galetti T, Tostain J, Cindolo L, Novara G, Zigeuner R, Bratti E, et al: Proposal for revision of the TNM classification system for renal cell carcinoma. Cancer 104: 2116-2123, 2005.

5. Yan M, Zhang L, Wu Y, Gao L, Yang W, Li J, Chen Y and Jin X: Increased expression of kindlin-2 is correlated with hematogenous metastasis and poor prognosis in patients with clear cell renal cell carcinoma. FEBS Open Bio 6: 660-665, 2016.

6. Soultati A, Stares M, Swanton C, Larkin J and Turajlic S: How should clinicians address intratumour heterogeneity in clear cell renal cell carcinoma? Curr Opin Urol 25: 358-366, 2015.

7. Kalvakolanu DV: The GRIMs: A new interface between cell death regulation and interferon/retinoid induced growth suppression. Cytokine Growth Factor Rev 15: 169-194, 2004.

8. Chidambaram NV, Angell JE, Ling W, Hofmann ER and Kalvakolanu DV: Chromosomal localization of human GRIM-19, a novel IFN-beta and retinoic acid-activated regulator of cell death. J Interferon Cytokine Res 20: 661-665, 2000.

9. $\mathrm{Lu} \mathrm{H}$ and Cao X: GRIM-19 is essential for maintenance of mitochondrial membrane potential. Mol Biol Cell 19: 1893-1902, 2008

10. Fearnley IM, Carroll J, Shannon RJ, Runswick MJ, Walker JE and Hirst J: GRIM-19, a cell death regulatory gene product, is a subunit of bovine mitochondrial NADH:ubiquinone oxidoreductase (complex I). J Biol Chem 276: 38345-38348, 2001.

11. Fan XY, Jiang ZF, Cai L and Liu RY: Expression and clinical significance of GRIM-19 in lung cancer. Med Oncol 29: 3183-3189, 2012

12. Zhang Y, Hao H, Zhao S, Liu Q, Yuan Q, Ni S, Wang F, Liu S, Wang L and Hao A: Downregulation of GRIM-19 promotes growth and migration of human glioma cells. Cancer Sci 102: 1991-1999, 2011.

13. Zhou Y, Li M, Wei Y, Feng D, Peng C, Weng H, Ma Y, Bao L, Nallar S, Kalakonda S, et al: Down-regulation of GRIM-19 expression is associated with hyperactivation of STAT3-induced gene expression and tumor growth in human cervical cancers. J Interferon Cytokine Res 29: 695-703, 2009.
14. Angell JE, Lindner DJ, Shapiro PS, Hofmann ER and Kalvakolanu DV: Identification of GRIM-19, a novel cell death-regulatory gene induced by the interferon-beta and retinoic acid combination, using a genetic approach. J Biol Chem 275: 33416-33426, 2000.

15. Li F, Ren W, Zhao Y, Fu Z, Ji Y,Zhu Y and Qin C: Downregulation of GRIM-19 is associated with hyperactivation of p-STAT3 in hepatocellular carcinoma. Med Oncol 29: 3046-3054, 2012.

16. Kong D, Zhao L, Du Y, He P, Zou Y, Yang L, Sun L, Wang H, Xu D, Meng X, et al: Overexpression of GRIM-19, a mitochondrial respiratory chain complex I protein, suppresses hepatocellular carcinoma growth. Int J Clin Exp Pathol 7: 7497-7507, 2014.

17. Bu X, Zhao C, Wang W and Zhang N: GRIM-19 inhibits the STAT3 signaling pathway and sensitizes gastric cancer cells to radiation. Gene 512: 198-205, 2013.

18. Bezhanova SD: Tumors of the kidney. The new 2016 WHO classification of tumors of the genitourinary system. Arkh Patol 79: 48-52, 2017 (In Russian).

19. Livak KJ and Schmittgen TD: Analysis of relative gene expression data using real-time quantitative PCR and the $2-\Delta \Delta C T$ method Methods 25: 402-408, 2001.

20. Xu L and Yang W: Criteria for judging the results of immunohistochemistry. Chin Oncol 6: 3, 1996.

21. Alchanati I, Nallar SC, Sun P, Gao L, Hu J, Stein A, Yakirevich E, Konforty D, Alroy I, Zhao X, et al: A proteomic analysis reveals the loss of expression of the cell death regulatory gene GRIM-19 in human renal cell carcinomas. Oncogene 25: 7138-7147, 2006.

22. Cui L, Meng Q, Wen J, YanZ, GaoZ, Tian Y, Xu P, Lian Pand Yu H. The effect of a gene associated with retinoid-interferon-induced mortality 19 (GRIM-19) on STAT3-induced gene expression in renal carcinoma. J Biochem 164: 285-294, 2018.

23. Hao M, Shu Z, Sun H, Sun R, Wang Y, Liu T, Ji D and Cong X: GRIM-19 expression is a potent prognostic marker in colorectal cancer. Hum Pathol 46: 1815-1820, 2015.

24. Shao YP, Cheng XD and Wan XY: Expression and clinical significance of GRIM-19 in epithelial ovarian carcinoma. Zhonghua Fu Chan Ke Za Zhi 47: 751-755, 2012 (In Chinese).

25. Zhou T, Chao L, Rong G, Wang C, Ma R and Wang X Down-regulation of GRIM-19 is associated with STAT3 overexpression in breast carcinomas. Hum Pathol 44: 1773-1779, 2013 